\title{
Interculturalidad en los procesos de formación profesional en Educación Física en Colombia
}

\author{
Interculturality in the Processes of Professional Training in Physical \\ Education in Colombia
}

Arnulfo Hurtado-Cerón ${ }^{1}$, Víctor Molina-Bedoya², Lida Osorio-Linares ${ }^{3}$

\begin{abstract}
RESUMEN
Este artículo expone los resultados de un trabajo de investigación que tuvo como objetivo identificar la presencia de la cultura de los pueblos indígenas y de la interculturalidad en los procesos de formación profesional en Educación Física en Colombia ${ }^{4}$. A nivel metodológico, la pesquisa se desenvolvió en el pensar en espiral, buscando la mau jxijunga (comprensión) y la mau jxiju' (interpretación), en un diálogo horizontal con la técnica del análisis de contenido para inferir y desocultar lo latente en las mallas curriculares, con apoyo de entrevistas a directores de programas del campo de formación profesional estudiado. Como resultados, se destacan el análisis de 46 programas del nivel de pregrado entre licenciaturas y profesionalizaciones y 15 de posgrados. Respecto a las unidades de relevancia de la investigación, solo se encontró un descriptor referido a la interculturalidad y ninguno a la cultura de los pueblos indígenas. Se concluye que existe una disonancia entre este panorama y el marco normativo colombiano que, de acuerdo con la Constitución Política de 1991, hace referencia a la necesidad de una cultura, una política pública y una educación comprometida con el rescate y la promoción de la diversidad para una sociedad que se reconoce multicultural y pluriétnica.
\end{abstract}

Palabras claves: cultura de los pueblos indígenas; diversidad cultural; formación profesional; educación física; estrategias pedagógicas.

\section{ABSTRACT}

This article presents the results of a research project that was aimed to identify the presence of the culture of indigenous peoples and interculturality in the processes of professional training in Physical Education in Colombia. On a methodological level, the research was carried out in spiral thinking,

\footnotetext{
${ }^{1}$ Profesor catedrático, Universidad de Antioquia, Antioquia, Colombia; arnulfo.hurtado@udea.edu.co.

2 Profesor titular, Universidad de Antioquia, Antioquia, Colombia; victor.molina@udea.edu.co.

3 Estudiante de Licenciatura en Educación Física, Universidad de Antioquia, Antioquia, Colombia; lidam.osorio@udea.edu.co.

${ }^{4}$ Esta indagación contó con el apoyo académico y financiero del grupo de investigación “Ocio, expresiones motrices y sociedad” del Instituto de Educación Física de la Universidad de Antioquia, Colombia.
} 
seeking the mau jxijunga (comprehension) and the mau jxiju' (interpretation), in a horizontal dialogue with the technique of content analysis to infer and uncover the latent, the not apparent, in the curricular meshes, with support of interviews with programs directors of the professional training field studied. As results, the analysis of 46 undergraduate programs and 15 postgraduate programs. Regarding the units of relevance of the research, only one descriptor was found referring to interculturality and none to the culture of the indigenous peoples. This allows to conclude that there is lack of concordance between this panorama and the Colombian regulatory framework that, according to the Political Constitution of 1991, refers to the need for a culture, a public policy and an education committed to the rescue and promotion of diversity for a society that recognizes itself as multicultural and multiethnic.

Keywords: Culture of indigenous people; cultural diversity; professional training; physical education; pedagogical strategies. 
INTERCULTURALIDAD EN LOS PROCESOS DE FORMACIÓN PROFESIONAL EN EDUCACIÓN FÍSICA EN

COLOMBIA / HURTADO-CERÓN, MOLINA-BEDOYA, OSORIO-LINARES

\section{Introducción}

Desde el año 1492 los pueblos originarios han tenido que transitar por grandes procesos de resistencia para poder "re-existir" y revivir la diversidad cultural que el poder imperante pretendió reducir por la vía de homogenizar la cultura bajo una sola idea de nación. En Colombia, en los años 70, se inician los procesos de refortalecimiento de las comunidades y organizaciones indígenas, en el marco de luchas que llevaron al reconocimiento de la pluriculturalidad en la Constitución Política de 1991. En este período, la interculturalidad, el multiculturalismo y la transculturalidad ganan cada vez mayor presencia en los debates académicos y sociales que se dan en la sociedad colombiana como expresión de un interés creciente por afianzar el carácter diverso del país y por favorecer procesos de convivencia más respetuosos de las diferencias, es decir, más democráticos.

Esta indagación encuentra resonancia en los ejercicios investigativos realizados por colegas de América Latina que han buscado problematizar los currículos profesionalizantes del campo, intentando develar sus lógicas constitutivas, así como examinar sus aportes o compromisos con temas de debate actual alrededor de primera infancia, género, discapacidad, interculturalidad, formación docente, producción de conocimiento y ocio (Silva y Molina, 2017).

Estudios que constatan la incipiente o baja presencia de discursos relacionados con la perspectiva intercultural o de reconocimiento de la diversidad social existente, tanto en América Latina como en Colombia.

Siendo Colombia un país pluricultural, es necesario llevar a cabo este trabajo investigativo para analizar la interculturalidad y la asunción de discursos y prácticas coherentes con las culturas de los pueblos indígenas, y de esta manera constatar si los currículos de las instituciones de Educación Superior que forman profesionales en el campo de la Educación Física, propician la generación de una cultura del respeto a la diversidad realmente existente o si por el contrario, hacen parte del sistema global que subalterniza e invisibiliza la existencia de esa amplia diversidad social que caracteriza a la nación colombiana. 
Desde estos estudios, se ha entendido que el campo de la Educación Física representa una construcción social importante en la que se constatan las disputas y las tensiones entre discurso, prácticas y experiencias históricas hegemónicas y de resistencia para la edificación de la "sociedad otra mejor posible". Así, la presencia o ausencia de determinados tópicos da cuenta del carácter incluyente o excluyente de los currículos formativos, para el caso, de la Educación Física como campo de saber y de poder.

Tal como lo señala el profesor Valter Bracht en el prefacio del libro "Formación profesional en Educación Física en América Latina. Currículos y horizontes", es necesario un reconocimiento de las particularidades de los territorios para desde allí facilitar procesos de relacionamiento académicos, profesionalizantes y culturales del sector, siempre sobre la base de una integración soberana y autónoma y en contra de prácticas integrativas subordinadas o sumisas (Silva y Molina, 2015).

Las categorías centrales del presente estudio fueron las siguientes: cultura de los pueblos indígenas, interculturalidad y formación profesional en educación física. "Cultura de los pueblos" se entendió, desde Geertz (1989), como el conjunto de esquemas, conductas, costumbres, usanzas, tradiciones y hábitos que yacen en el proceso histórico de las expresiones del ser humano. Es decir, "cultura de los pueblos" sería el tejido social que entrama cosmovisiones, prácticas, epistemologías, tradiciones, ancestralidades, características y rasgos propios construidos en la historicidad, y que hace que los pueblos se distingan de otros grupos sociales dando forma a la diversidad y diferencia cultural.

Por "interculturalidad" se comprendió el proceso de diálogo entre-cultural de pueblos indígenas, campesinos, mestizos, afros, gitanos, entre otros. Este concepto alude al establecimiento de relaciones de convivencia, comunicación entre las diversas epistemologías, respeto por la otredad, unidad política, económica y social para promover la diversidad y el enriquecerse de todos (Villodre, 2012).

Por último, la "formación profesional en Educación Física" se definió como el proceso por el cual un individuo se capacita en el campo de conocimiento específico para desenvolverse en temas de interés empírico, técnico y científico, para el saber y el hacer en diferentes contextos sociales. En términos metodológicos, esto incluye habilidades 
INTERCULTURALIDAD EN LOS PROCESOS DE FORMACIÓN PROFESIONAL EN EDUCACIÓN FÍSICA EN

COLOMBIA / HURTADO-CERÓN, MOLINA-BEDOYA, OSORIO-LINARES

comunicativas, de interacción, toma de decisiones y asunción de responsabilidades sociales (Maura y Tirados, 2008).

\section{Metodología}

Con el propósito de abordar el objetivo específico de examinar las estrategias implementadas por los currículos de formación profesional en Educación Física para abordar la diversidad social y cultural del país, se recurrió a la identificación de directivos de universidades públicas y privadas, a los cuales se les entrevistó con un derrotero de preguntas abiertas emergentes de la fase previa de análisis de contenido tanto de los documentos institucionales de las instituciones formadoras como del marco normativo del campo de la Educación Física en Colombia.

Se aplicaron dos entrevistas: la primera a un directivo de institución pública y la segunda a un líder de universidad privada. El criterio que se tuvo en cuenta fue intencional de acuerdo tanto a la factibilidad de acceso como de disponibilidad para participar en la entrevista. Estas entrevistas fueron grabadas en grabadora de audio (Sony ICD-P 320) y luego transcritas, analizadas y codificadas.

La “entrevista abierta”, según Ileana Vargas (2012), es una técnica de la investigación cualitativa que se emplea para la recolección de información sin categorías preestablecidas. Por lo tanto, la secuencia de preguntas no se prefija. Las preguntas son flexibles para que haya una mayor adaptación a las necesidades de la pesquisa y de las características de los sujetos, y de esta forma permitan acceder a las percepciones, las creencias, las opiniones, los significados y las actitudes de las experiencias de las personas.

Esta investigación se realizó desde la perspectiva epistemológica del diálogo y la ecología de saberes. Es así que se partió desde el "enraizamiento del ombligo en la madre tierra” para

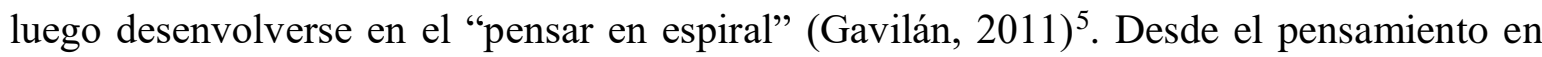

\footnotetext{
${ }^{5}$ La espiral representa el desenvolvimiento de la existencia del pueblo Nasa, que hace posible desenvolver un pensamiento circular que nos permite avanzar y estar retornando al origen. En esta cosmovisión, el ombligo
} 
REVISTA SABERES EDUCATIVOS, Nº 4, ENERO-JUNIO 2020

espiral se buscó la mau jxijunga (comprensión) y la mau jxiju' (interpretación) de la realidad y las situaciones socioculturales referidas a la cultura de los pueblos indígenas y la interculturalidad en los escenarios de las instituciones de educación superior. Esta representación metodológica da cuenta del proceso como se llegó a esta indagación, puesto que para el pueblo ancestral Nasa, el mundo constantemente refleja acaeceres (fenómenos) que se desenvuelven de forma circular y que van imbricados con otros desde el origen de la vida (Figura 1).

\section{Figura 1}

Investigación en la espiral

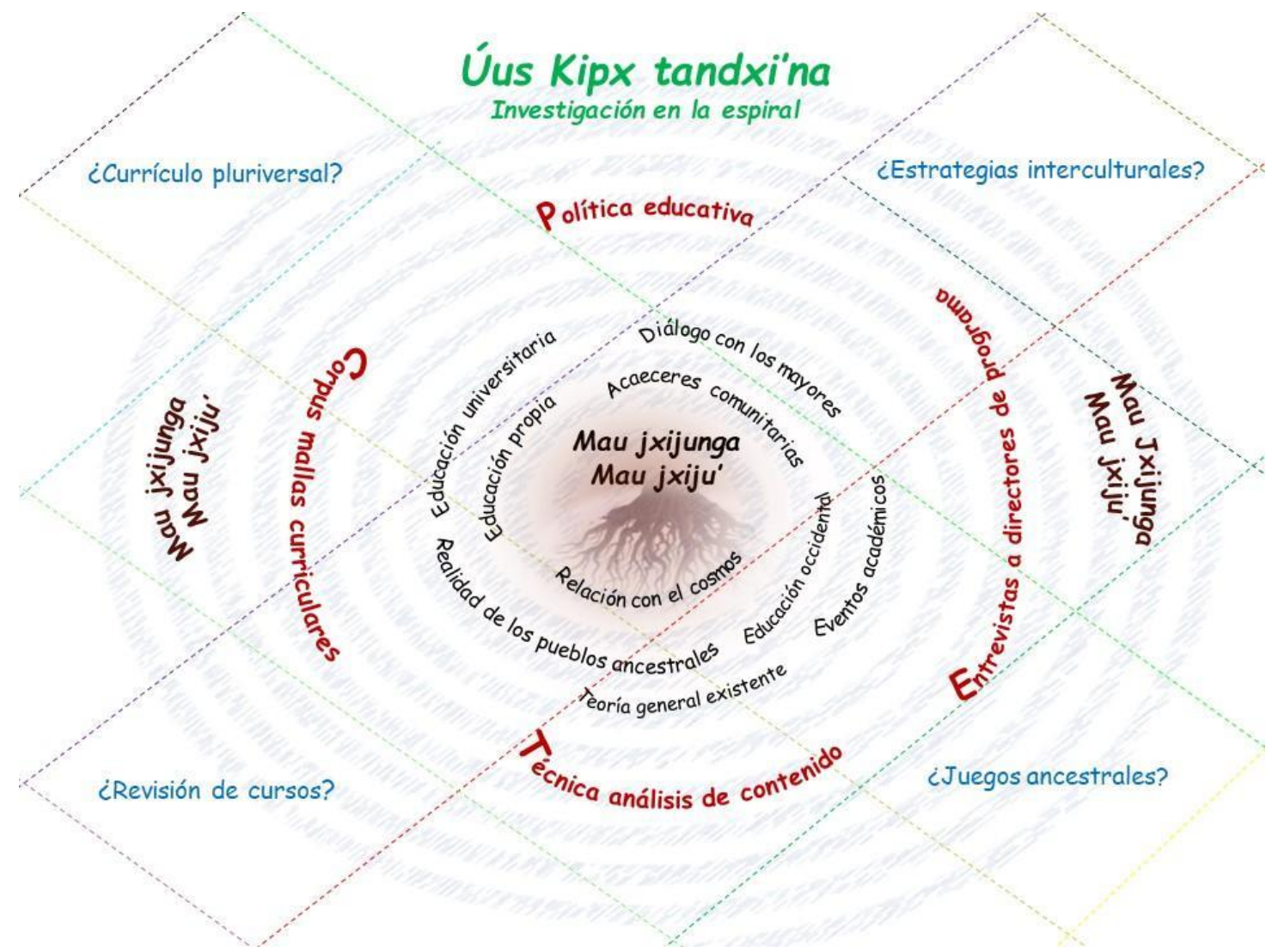

comienza a tejer la vida conectada con la madre tierra, comprendiendo (Mau jxijunga) e interpretando (Mau jxiju) el mundo. 
INTERCULTURALIDAD EN LOS PROCESOS DE FORMACIÓN PROFESIONAL EN EDUCACIÓN FÍSICA EN

COLOMBIA / HURTADO-CERÓN, MOLINA-BEDOYA, OSORIO-LINARES

El caminar en espiral se tejió de forma horizontal con la "técnica del análisis de contenido" (Bardin, 1986) para inferir y develar lo latente, lo no aparente en los documentos institucionales y mallas curriculares de las universidades que ofertan programas de formación profesional en el área en Colombia, tanto de nivel de pregrado como de posgrado (Corona y Kaltmeier, 2012).

Como técnica investigativa, el análisis de contenido se ocupa de las expresiones verbales consignadas en los textos, pero en esencia no se remite a ellos en sí mismos, sino a algo en relación con lo cual estos funcionan en cierto modo como instrumento, para centrarse propiamente en el análisis de lo contenido fuera de ellos. Es "un procedimiento destinado a desestabilizar la inteligibilidad inmediata de la superficie textual, mostrando sus aspectos no directamente intuibles y, sin embargo, presentes" (Delgado, 1998, p.182).

La estrategia de análisis fue extensiva, es decir, se trabajó con un corpus relativamente amplio representado por las mallas y los documentos institucionales elaborados por diversos autores e instituciones. La estrategia intertextual que se privilegió fue la del método discriminatorio, desde la cual se conformaron conjuntos de textos según las fuentes de producción, en este caso documentos de las páginas virtuales de las universidades y mallas curriculares de la plataforma Sistema Nacional de Información de la Educación Superior del Ministerio de Educación Nacional (SNIES del MEN).

Para la fase de análisis se consideró como unidades básicas de relevancia la interculturalidad y la cultura de los pueblos indígenas, descriptores que para Delgado representan las "unidades de registro" (1998). Estas unidades de registro se localizaron en las unidades de contexto y fueron relacionadas con la información extratextual, dando paso a la fase de codificación, en la cual las unidades de registro fueron relacionadas con sus respectivas unidades de contexto.

Luego de esta codificación, las unidades de registro fueron contabilizadas y relacionadas, extrayendo información referida a la presencia o ausencia de cada una de ellas, así como frecuencia e intensidad de las mismas. Seguidamente se pasó a la fase de categorización, que permitió la clasificación de las unidades de registro según similitudes y diferencias posibles, de acuerdo a criterios definidos previamente por la investigación, para luego establecer 
esquemas categoriales. Por último, se dio paso a la fase interpretativa e inferencial, que representó propiamente el momento teórico de la indagación y dio cuenta, básicamente, de las realidades subyacentes a la producción de esos datos (Delgado, 1998).

\section{Resultados}

\section{Interculturalidad y cultura de los pueblos indígenas en las mallas curriculares}

Al examinar las mallas curriculares de los programas de formación, no se encontraron elementos directos que dieran cuenta de la forma como se abordan los componentes objeto de este estudio: interculturalidad y cultura de los pueblos indígenas en el campo de la Educación Física.

No obstante, en los 46 planes de estudio del nivel de pregrado se hallaron 66 unidades de registro asociadas o relacionadas con el objeto de la investigación, repartidas en cuatro bloques. El primero de ellos da cuenta de 31 unidades de registro que asocian la cultura con lo social, lo político, las prácticas corporales, el desarrollo humano, la educación, la pedagogía y lo comunitario, entre otros aspectos (tabla 1), descriptores que pueden tener cercanía a la diversidad sociocultural y a la pluriculturalidad. El segundo bloque pondera el concepto de cultura física enfocada al deporte, a la administración, a la biología, a la recreación y a la pedagogía, con un total de 23 unidades. En el tercer bloque se registraron 8 unidades de relevancia relacionadas con la danza folclórica y los juegos tradicionales. Y en un último bloque se ubicaron 4 unidades de registro que resaltan la diversidad, la antropología y la cosmovisión. 
INTERCULTURALIDAD EN LOS PROCESOS DE FORMACIÓN PROFESIONAL EN EDUCACIÓN FÍSICA EN

COLOMBIA / HURTADO-CERÓN, MOLINA-BEDOYA, OSORIO-LINARES

\section{Tabla 1}

Interculturalidad y cultura de los pueblos indígenas en pregrados

Unidades de Registro asociadas a lo sociocultural
Unidades de Registro asociadas a la cultura física
Unidades de Registro asociadas a la

Educación Física de los pueblos indígenas
Unidades de Registro asociadas a la cultura de los pueblos indígenas
Hombre cultura, sociedad y naturaleza

Hombre, cultura y sociedad

Desarrollo humano, social y cultural

Construcción sociocultural del cuerpo

Cuerpo, sociedad, cultura y procesos de humanización

Lenguaje, sociedad y cultura

Cultura, sociedad y prácticas corporales

Experiencias

corporales

socioculturales

Experiencias corporales y sus relaciones con la cultura y el poder

Imaginarios socioculturales de la Educación Física y sus implicaciones en el desarrollo del potencial humano
Fundamentos de la cultura física

Administración y legislación de la cultura física

Construcción cultural del cuerpo

Teoría y epistemología de la cultura física

$$
\text { Gestión y }
$$
administración de la cultura física ambiental

TIC aplicadas a la cultura física

Didáctica de la cultura física

Investigación y cultura física recreativa $\mathrm{y}$ deportiva

Procesos evaluativos en cultura física, recreación y deporte

Estadísticas aplicadas a la
Didáctica del juego y del juego tradicional

Lúdica tradicional oral y cultural

Didáctica de los juegos
tradicionales

Folklore

colombiano

Danza folclórica

Danza popular y tradicional

Danza y folclor
Educación y diversidad

Educación en y para la diversidad

Inclusión en contextos diversos

Antropología y cosmovisiones 
REVISTA SABERES EDUCATIVOS, N 4, ENERO-JUNIO 2020

\begin{tabular}{|c|c|}
\hline $\begin{array}{l}\text { Sociedad, cultura y } \\
\text { educación }\end{array}$ & $\begin{array}{c}\text { cultura física y } \\
\text { deportiva }\end{array}$ \\
\hline $\begin{array}{l}\text { Didáctica del } \\
\text { deporte comunitario }\end{array}$ & $\begin{array}{l}\text { Investigación y } \\
\text { cultura física }\end{array}$ \\
\hline $\begin{array}{l}\text { Educación cultural y } \\
\text { sociedad }\end{array}$ & $\begin{array}{l}\text { recreativa y } \\
\text { deportiva II }\end{array}$ \\
\hline $\begin{array}{l}\text { Construcción social } \\
\text { del sujeto }\end{array}$ & $\begin{array}{c}\text { Cultura física y } \\
\text { discapacidad }\end{array}$ \\
\hline $\begin{array}{l}\text { Humanidad, } \\
\text { universidad y cultura }\end{array}$ & $\begin{array}{c}\text { Introducción a la } \\
\text { cultura física }\end{array}$ \\
\hline $\begin{array}{l}\text { Educación, } \\
\text { comunicación y }\end{array}$ & $\begin{array}{c}\text { Cultura física } \\
\text { deportiva }\end{array}$ \\
\hline cultura & $\begin{array}{l}\text { Historia de la } \\
\text { cultura física }\end{array}$ \\
\hline Diversidad cultural y & \\
\hline TIECs & $\begin{array}{l}\text { Cultura física } \\
\text { especial (TP) }\end{array}$ \\
\hline Cultura política & \\
\hline Cultura religiosa & $\begin{array}{l}\text { Fundamentos de } \\
\text { biología humana }\end{array}$ \\
\hline $\begin{array}{l}\text { Sociedad, cultura y } \\
\text { desarrollo }\end{array}$ & $\begin{array}{l}\text { aplicada a la } \\
\text { cultura física }\end{array}$ \\
\hline $\begin{array}{l}\text { Antropología de la } \\
\text { Educación Física y } \\
\text { el deporte }\end{array}$ & $\begin{array}{c}\text { Marco legal de la } \\
\text { cultura física en } \\
\text { Colombia }\end{array}$ \\
\hline $\begin{array}{l}\text { Introducción a la } \\
\text { cultura }\end{array}$ & $\begin{array}{l}\text { Diseño y gestión } \\
\text { de proyectos de la } \\
\text { cultura física }\end{array}$ \\
\hline $\begin{array}{l}\text { Antropología } \\
\text { cultural }\end{array}$ & $\begin{array}{l}\text { Pedagogía de la } \\
\text { cultura fisca en el }\end{array}$ \\
\hline Socioantropología & adulto mayor \\
\hline $\begin{array}{l}\text { Cultura y } \\
\text { comunicación }\end{array}$ & Cultura física \\
\hline
\end{tabular}

Semiótica de la

cultura

Corporeidad y

cultura

Antropología

cultural 
Cultura

emprendedora

Cultura tecnológica

Cultura humanística

De las 46 mallas curriculares analizadas, las unidades de registro asociadas se ubicaron en 27 planes de estudio. En primer lugar, los descriptores relacionados a la sociocultura destacan en la Universidad Pedagógica Nacional (10); en segundo lugar, en las instituciones de educación superior Corporación Universitaria Cenda (3) y la Universidad San Buenaventura de Medellín (3); en tercer orden, en la Universidad San Buenaventura de Cartagena (2) y en la Universidad del Cauca (2); y, por último, se distribuyen en igual proporción de unidades de relevancia en 11 universidades del país (1) (Figura 2).

Respecto a las unidades de relevancia afines a la cultura física, en primer orden se ubica la Universidad del Atlántico (7), seguidamente las universidades Santo Tomas (4) e INCCA de Colombia (3), y entre 1 y 2 descriptores se encuentran 5 instituciones de educación superior.

Seguidamente, ubicándonos en el descriptor que hace referencia a la educación física de los pueblos indígenas, en primer lugar, se halla la Universidad del Tolima (2) y las demás universidades con (1) mención.

Por último, frente a la categoría cultura de los pueblos indígenas, se encontró (1) referencia en cada una de las siguientes instituciones: Fundación Universitaria Luis Amigó, Universidad de Pamplona, Universidad del Cauca y Universidad San Buenaventura de Cartagena. 
REVISTA SABERES EDUCATIVOS, No 4, ENERO-JUNIO 2020

Figura 2

Distribución de unidades de registro según universidades

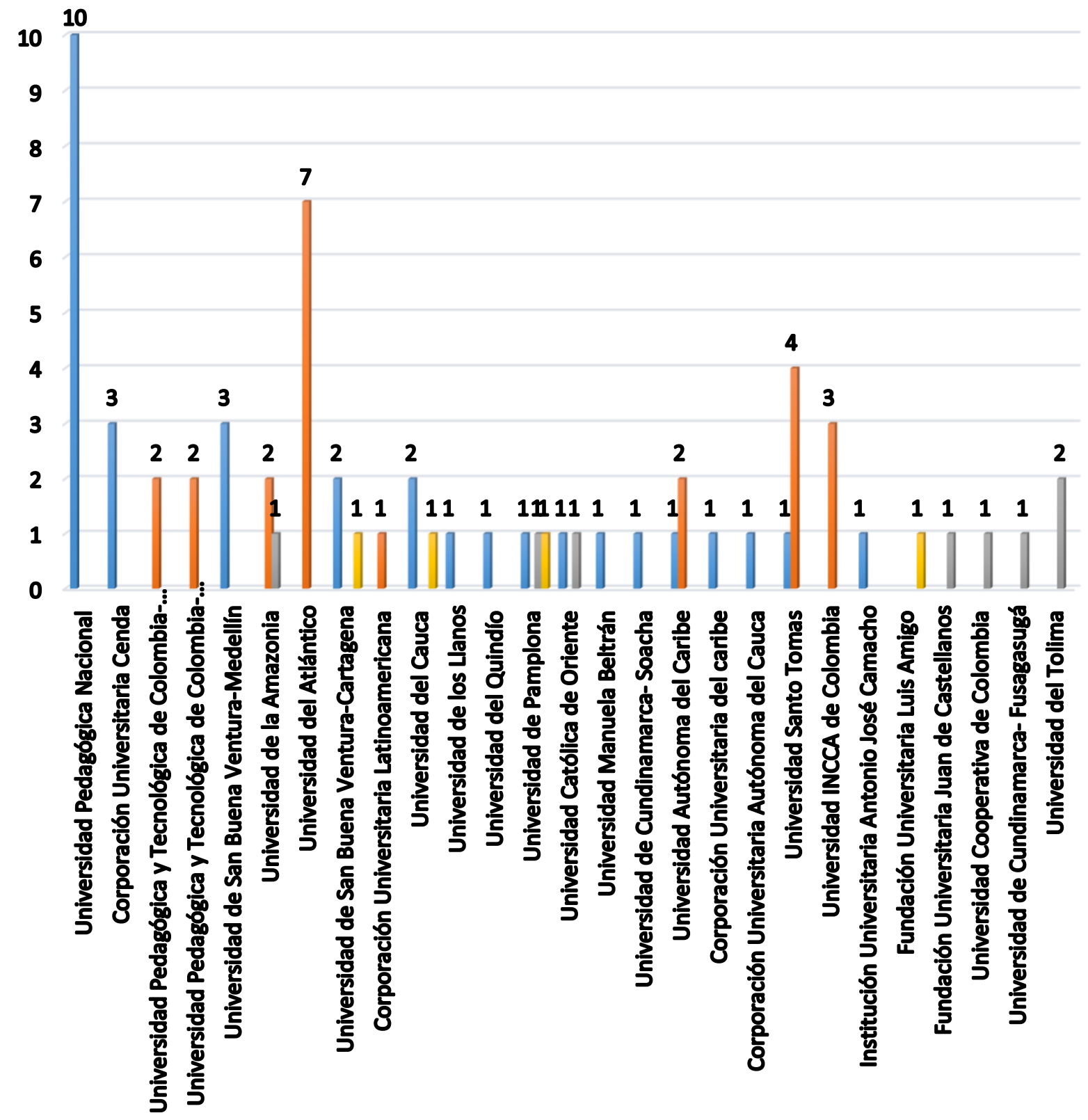

A LA SOCIOCULTURA

A LA CULTURA FÍSICA

- A LA EDUCACIÓN FÍSICA DE LOS PUEBLOS INDIGENAS

A LA CULTURA DE LOS PUEBLOS INDIGENAS 
INTERCULTURALIDAD EN LOS PROCESOS DE FORMACIÓN PROFESIONAL EN EDUCACIÓN FÍSICA EN

COLOMBIA / HURTADO-CERÓN, MOLINA-BEDOYA, OSORIO-LINARES

En el nivel de posgrado, en la Universidad de Antioquia (sede Medellín y Apartado) y en la Universidad de los Llanos se hallaron 4 unidades de registro que referencian la cultura relacionada con lo somático, la sociedad y la pedagogía en 15 currículos (8 maestrías y 7 especializaciones).

No se observó en este nivel interés por problematizar asuntos alrededor de lo sociocultural, la diversidad, la cultura de los pueblos indígenas y la interculturalidad.

\section{Estrategias institucionales para la interculturalidad}

Respecto a las estrategias pedagógicas que emplean las instituciones formadoras en Educación Física para abordar los temas de la cultura de los pueblos indígenas y la diversidad social, no se encontró claridad en la forma de su concreción como intenciones o contenidos curriculares que evidencien su articulación al direccionamiento pedagógico y político definido desde la Constitución Política. Al respecto se puede leer en una de la entrevista a un directivo de programa:

(...) las normas a veces son muy genéricas. Entonces cuando dicen vamos a trabajar la interculturalidad, la diversidad, cierto, ahí está expuesto, pero para todo el mundo, para todo el que la lee no es claro qué es interculturalidad, de qué hablan, a qué se refiere cuando hablan de diversidad de las comunidades indígenas y, entonces, ahí es donde se distorsiona un poco la normativa, la política (...). (E. D. U. Pu, 2017)

Como se aprecia en la cita anterior, al no tener conocimiento de lo expuesto en los marcos normativos, y al ser imprecisas las nociones y los conceptos, resulta problemático definir las estrategias que permitan su abordaje en los planes de estudio de la Educación Física.

En la misma dirección se observa poca preocupación por profundizar lo establecido en la norma respecto a los temas de diversidad social:

(...) no es que sea un ducho en la Constitución, tengo que aceptar que también estoy hablando desde cierta ignorancia de ella, cierto... y, usualmente, cuando se mencionan en la Constitución, se mencionan como un patrimonio cultural, como si fueran un 
objeto de referencia histórica para ser lucido ante comunidades internacionales, cierto, pero se preserva como patrimonio cultural, como si fuera un objeto, como si fuera una cosa que hay que tener ahí. (E. D. U. Pr, 2017)

Por otra parte, aunque no se interprete la norma en los debates interinstitucionales hay preocupación por dar lugar a la cultura de los pueblos indígenas, la diversidad social e intercultural. Según las palabras de un directivo,

(...) en cada uno de los encuentros que hacemos con ARCOFADER encontramos que eso siempre sale a la luz y que se le está dando realmente relevancia (...). Y una cosa muy interesante en ese debate y en esa discusión es cómo conservar esas costumbres y esa idiosincrasia de los pueblos indígenas porque no se trata de que los procesos educativos derrumben todos esos elementos de la tradición que cada uno de los grupos tienen de acuerdo a las poblaciones donde se ubican. (E. D. U. Pu, 2017)

De la misma manera, en la entrevista al director de universidad privada de corte confesional destaca el ofrecimiento de dos cursos en el nivel de pregrado y posgrado que desarrollan contenidos alusivos a la interculturalidad, los grupos étnicos y la realidad social desde una perspectiva socio crítica:

(...) en sus asignaturas tienen una que se titula 'Necesidades de educación especial' y otra que llama 'Educación para jóvenes y adultos', y en esa 'Educación para jóvenes y adultos' se habla de la temática de otras educaciones y en esa temática de otras educaciones se tocan ese tipo de particularidades, cuando se quiere hablar de una pedagogía en contexto... de un contexto socio crítico. Entonces, la mayoría de los docentes... hay, digamos, unos tres o cuatro docentes que manejan el área pedagógica (...) de culturas indígenas y siempre sazonan los contenidos temáticos con eso, buscando esas pedagogías, esos saberes contextualizados en esas comunidades (...). (E. A. D. U. Pr, 2017)

Aunque los nombres de los cursos no estén relacionados directamente con las unidades de registro, el director del Programa de Educación Física de la Universidad Católica de Oriente (UCO), (C. M. C. I, 2016), asegura abordar estos temas en las asignaturas mencionadas. Así mismo, es posible observar en la Universidad San Buenaventura, en la Santo Tomas y en la 
INTERCULTURALIDAD EN LOS PROCESOS DE FORMACIÓN PROFESIONAL EN EDUCACIÓN FÍSICA EN

COLOMBIA / HURTADO-CERÓN, MOLINA-BEDOYA, OSORIO-LINARES

Fundación Luis Amigó (C. M. C. I, 2016) temas en sus planes de estudio que indirectamente tienen relación con la multiculturalidad y lo étnico.

Desde los direccionamientos administrativos de la UCO se menciona la realización de un evento intercultural cada año para recrear diálogos entre las comunidades invitadas, tales como los Wayúu y Embera. En este evento se comparten experiencias con la población estudiantil en los asuntos de hábitat, educación, problemáticas socioculturales, perspectivas de vida, tradiciones filosóficas, bailes típicos, juegos tradicionales, tiempo libre, gastronomía y artesanías. Este evento tiene una duración de tres días y es organizado por la Facultad de Comunicación Social, y en él también participa, como parte de la comunidad académica, el Programa de Educación Física.

Respecto al Programa de Educación Física de la UCO, este no contempla, de manera específica, ninguna estrategia pedagógica para promover el encuentro intercultural entre los estudiantes indígenas, afros, negritudes, gitanos y otros. Sin embargo, el director del Programa considera importante abordarlo desde una pedagogía crítica, en la idea de visibilizar pueblos que han sido ignorados por el capitalismo desde los tiempos de la colonización.

En el discurso de la directiva de la universidad pública, esta señala que no hay una asignatura específica para asumir la diversidad, la cultura de los pueblos indígenas, la sociocultura y la interculturalidad. Sin embargo, dice tener conocimiento de procesos pedagógico-educativos con comunidades indígenas y afros, adquirido durante su labor profesional, que considera idóneo para viabilizar procesos estratégicos en la dependencia que dirige.

(...) mi proceso formativo tuvo mucho que ver con (...) la cultura, con el arte, la diversidad étnica en todas las regiones de Colombia, entonces, yo siempre he sido una abanderada de que esos procesos se vivencien distintos y que las personas que han llegado al Instituto, específicamente a esos trabajos, tengamos como un acercamiento distinto (...). (E. A. D. U. Pu, 2017) 
Esta directiva considera como estrategia pedagógico-educativa la individualización de la enseñanza con los estudiantes que pertenecen a los pueblos étnicos. Este discurso plantea algunos problemas puesto que, a nuestro modo, si consideramos que se deberían establecer procesos educativos interculturales visibilizando los diversos conocimientos que poseen las variadas culturas.

En el mismo orden, considera que en los cursos de práctica pedagógica y contexto social se desenvuelven los temas que tocan la diversidad social del país de manera transversal, mediante la libertad de cátedra de cada docente. Reitera, eso sí, la falta de estrategias de integración intercultural de las comunidades indígenas, afros, negritudes y otras en la dependencia, aunque indica estar en proceso de organizar actividades integrativas en concordancia con la oficina de bienestar, pero no en la idea de orientar los currículos, sino para generar actividades de expresiones culturales entre la comunidad estudiantil presente en el Programa de Educación Física. Del mismo modo, considera que las iniciativas para la interculturalidad y la socialización de la diversidad sociocultural deben surgir del empoderamiento de los mismos estudiantes.

En definitiva, se destaca de los discursos de los/as directores/as-administrativos/as, tanto de universidad pública como privada, la ausencia de estrategias en los currículos para abordar la cultura de los pueblos indígenas y la diversidad social en los procesos de formación profesional en el campo de la Educación Física. Empero, se hallan algunas actividades interactivas que se celebran anualmente como espacio de encuentro intercultural, lideradas por las dependencias de la misma institución y dos cursos en los que se estarían asumiendo contenidos relativos al área. Así mismo, vale destacar que en la institución privada hay más iniciativas por hacer visible y dignificar los grupos étnicos en los procesos de educación superior.

Por último, en la universidad privada se reconoce la necesidad de emprender procesos educativos interculturales desde el respeto a la diferencia cultural, ya que se expresa ser testigo de algunos estudiantes indígenas que han ingresado al programa y al terminar los estudios no han querido regresar a las comunidades de origen, debido a la "captura", si así se puede llamar, que propician los procesos educativos eurocéntricos y el consecuente desarraigo de sus cosmovisiones. De tal forma, muchos han optado por quedarse en la ciudad 
INTERCULTURALIDAD EN LOS PROCESOS DE FORMACIÓN PROFESIONAL EN EDUCACIÓN FÍSICA EN

COLOMBIA / HURTADO-CERÓN, MOLINA-BEDOYA, OSORIO-LINARES

trabajando, a causa de la ausencia de diálogos interculturales que permitan mantener y fortalecer las culturas, tal como se direcciona desde la política pública del país.

\section{Discusión}

Los resultados de este estudio develan, desde el punto de vista de la formación superior o profesional en el campo de la Educación Física, la ausencia de la cultura de los pueblos indígenas y la interculturalidad, puesto que no se ubicó referencia directa que diera cuenta del objeto de este estudio. No obstante, en los planes de estudio se ubicaron descriptores asociados a la sociocultura, relacionados con lo político, lo comunitario, el desarrollo humano, la educación, la pedagogía, entre otros aspectos.

Las unidades de registro referidas a la cultura física, deportiva y de las TIC son las que más destacan. En otro nivel del análisis, se ubicaron dos bloques con pocas unidades de relevancia que relacionan la Educación Física con la cultura de los pueblos indígenas, es decir, temas que podrían estar cercanos a las prácticas lúdicas de las comunidades indígenas.

Este panorama es alarmante, puesto que los descriptores relacionados a lo sociocultural de manera general- para asumir la educación profesional de los pueblos indígenas, pueden estar inmersos en el mismo recorrido histórico que ha determinado la práctica universitaria eurocéntrica y afianzadora de modelos educativos universales basados en el pensamiento lineal-racional que invisibiliza las cosmovisiones y epistemologías de los diferentes pueblos (Sierra, 2004).

Un ejemplo de ello lo representa la Universidad de Antioquia, institución que acoge una diversidad de grupos étnicos procedentes de diferentes regiones del país como los Wayuu, Inga, Kamentza, Guambiano, Nasa, Afros, ente otros. Pueblos que, según Sierra (2004), son tratados como estudiantes universitarios en general, sometidos al modelo pedagógico autoritario y vertical que no da lugar a la participación desde la diferencia constitutiva de cada cultura. Se trata de una situación preocupante que debe trascender hacia "la decolonización del conocimiento y [...] la decolonización de las instituciones productoras o 
administradoras del conocimiento" (Castro, 2007, p.88), para que el diálogo de saberes sea posible.

En tal sentido, la posibilidad de un diálogo intercultural del conocimiento de los pueblos, con relación a las prácticas propias de socialización, en las que acaece lo lúdico, la recreación, los juegos ancestrales y el ocio son subalternizadas y ocultadas por las prácticas hegemónicas como las deportivas y las biomédicas.

Hay allí un interés por enfocar los procesos educativos al servicio de la lógica del mercado, al querer convertir el ejercicio físico en una suerte de cultura alrededor de lo físico y lo deportivo, como moda para satisfacer o promover las necesidades de consumo. En un estudio realizado en Brasil y Argentina por Bracht y Crisorio (2003) se subraya una crisis de identidad en el campo de la educación física en la modernidad occidental, expresada en procesos de desinstitucionalización y destradicionalización de las prácticas sociales por vía de las nuevas funciones que fija el sistema capitalista. El área tiende, de esta forma, a fundamentarse en la construcción de un universo simbólico orientado por el discurso biomédico-militar, más afín a la especificidad de la institución deportiva.

Se podría entender, así, por qué el tema de la interculturalidad no se encuentra para fortalecer la diversidad cultural en las instituciones de educación superior, pues el sistema imperante tiende a homogenizar las culturas para tener el control de la sociedad y así poner en marcha los proyectos transnacionales en vía de la extracción de los recursos, la cultura y la sabiduría presente en los territorios e instalar la idea del ciudadano consumidor universal.

Resulta problemático, en tal sentido, que un país como Colombia, que reconoce su carácter multicultural y pluriétnico -en su Constitución-, no evidencie esto en los diferentes ámbitos de la vida social e institucional, como garantía de respeto real y efectivo, de identidad étnica y preservación de las culturas y, más aún, como condición básica para construir una sociedad en paz a partir de la diferencia.

Por otra parte, las estrategias que direccionan las instituciones formadoras en Educación Física para abordar los temas de la cultura de los pueblos indígenas y la interculturalidad no son claras, pues en las entrevistas a los/as directivos/as de las universidades se constató el desconocimiento de la norma que orienta las políticas educativas relativas a estos temas. Esto 
INTERCULTURALIDAD EN LOS PROCESOS DE FORMACIÓN PROFESIONAL EN EDUCACIÓN FÍSICA EN

sucede, no obstante que la Constitución Política establece en su artículo 123 que "los servidores públicos están al servicio del Estado y de las comunidades; ejercerán sus funciones en la forma prevista por la Constitución, la Ley y el reglamento" (Constitución Política de Colombia de 1991, 2016, p. 62).

Para atender este mandato constitucional, la Ley de Educación Superior establece respecto a la defensa de las culturas existentes en Colombia, en sus artículos 2 y 4, que la educación es un servicio público, cultural, y esencial para promover la autonomía personal, la libertad de pensamiento en el pluralismo y en contribuir con la preservación de los saberes de las diferentes culturas del país (Ley 30, 1992).

En la norma específica que regula la actuación del campo de la educación física, la recreación y el deporte (Ley 181 de 1995) se encuentran lineamientos coherentes con lo enunciado anteriormente, en especial la referencia a fomentar las recreaciones autóctonas, folclóricas, tradicionales y las fiestas típicas, como expresiones culturales del territorio, y todos los actos que reafirmen la identidad nacional.

En igual dirección, la Serie Lineamientos Curriculares de la Educación Física, Recreación y Deporte (MEN, 2000) y el documento Orientaciones Pedagógicas (MEN, 2010) exhortan a la promoción del conocimiento de la diversidad, la culturalidad y la pluriculturalidad en los procesos curriculares del área, impulsando y refortaleciendo las prácticas tradicionales recreativas y deportivas para socializarlas y facilitar el diálogo con las diversas culturas de la nación.

Se entiende en este orden de ideas que las personas en roles administrativos y directivos universitarios tienen responsabilidad frente al cumplimiento de lo establecido en la Constitución y la ley, por tanto, deberían procurar alternativas reales y efectivas para el abordaje de estos asuntos en los currículos.

Por otra parte, desde una opción más de tipo ético y político, no reconocer la diversidad cultural es impedir que la riqueza multicultural sea posible, con todo lo que esto entraña para una sociedad heterogénea como la colombiana, tan necesitada de proyectos transculturales que permitan superar el racismo, el patriarcado, la exclusión y la discriminación. 
Una crítica estructural en este orden de cosas tiene que ver con las universidades actuales que imparten un conocimiento que consideran legítimo para todos, reproductora de modelos que se centran más en la unificación que en la promoción de la alteridad, la pluriversidad y el desarrollo de acciones educativas basadas en la otredad. Como se encontró en esta investigación, las universidades públicas y privadas están más orientadas, al parecer, a reproducir las lógicas de las políticas neoliberales, formando sujetos para las demandas de la sociedad de consumo y de espectáculo.

Es urgente, de acuerdo con lo anterior, promover diálogos interculturales entre las diversas poblaciones, sean estas indígenas, afros, gitanas, entre otras, en las universidades. Esto es claro para De Sousa (2010), quien plantea la necesidad de "un diálogo transcultural, pensado desde un intercambio no solo entre diferentes saberes, sino también entre diferentes culturas, es decir, entre universos de significados diferentes y en un sentido fuerte, inconmensurables" (p.72).

En todo caso, hay que tener cuidado con los procesos de folklorización de las culturas en el afán por atender asuntos del orden administrativo y legales, como se puede leer cuando los directivos procuran resolver este mandato por la vía de acciones o programas puntuales. Aquí la crítica de Sandoval (2016) adquiere relevancia:

Folklorizan la interculturalidad con desfiles, manifestaciones artísticas, conmemoración de fechas como el 'día de la raza', el 'día del encuentro de los dos mundos", el "día de los pueblos indígenas", el “día de la mujer indígena”, el "día de la diversidad", el "año nuevo indígena" etc. (p. 29)

Esta folklorización convierte a las culturas en una suerte de objeto cultural a ser consumido, más que una condición existencial necesaria para una vida en armonía a partir de lo diverso. Walsh (2008) reconoce que la interculturalidad aún no existe, debido a que es un proyecto en construcción de la posibilidad real de diálogo entre las culturas. Desde una perspectiva social y no únicamente económica, es necesaria la generación de espacios de encuentro, articulación y asociación entre culturas. 
INTERCULTURALIDAD EN LOS PROCESOS DE FORMACIÓN PROFESIONAL EN EDUCACIÓN FÍSICA EN

COLOMBIA / HURTADO-CERÓN, MOLINA-BEDOYA, OSORIO-LINARES

\section{Conclusiones}

La ausencia directa de los temas de la cultura de los pueblos indígenas y la interculturalidad en los procesos de formación profesional en el campo de la Educación Física en Colombia demanda una reflexión crítica por parte de las instituciones gubernamentales, tales como el Ministerio de Educación Nacional y las universidades con programas en el área para que procuren estrategias que posibiliten un diálogo más armonioso y respetuoso de la pluriculturalidad.

Las universidades, en especial, como ámbitos de circulación de saberes y experiencias, deben propiciar la multiplicidad de cosmogonías existentes desde enfoques transdiciplinares y transculturales para superar la homogeneidad que ignora encubre y desarraiga.

Se constató el desconocimiento de la norma que orienta las políticas educativas relativas a los pueblos étnicos. En tanto, se precisa de más compromiso por parte de los/as directores/as de programas del campo de la educación física, la recreación y el deporte para situar la diversidad y la pluriculturalidad, y para generar estrategias reales de asunción de las múltiples culturas que están presentes en las instituciones de educación superior, como lo disponen los diversos preceptos de Colombia.

También, se destaca en los resultados la ausencia de estrategias en los currículos para abordar la cultura de los pueblos indígenas y la diversidad social en los procesos de formación profesional en el campo de la Educación Física tanto de universidad pública como privada. Empero, se hallan algunas actividades interactivas que se celebran anualmente como espacio de encuentro intercultural, el cual podría estar cayendo en la folclorización de las culturas en el afán por atender asuntos del orden administrativo y legales, como se puede leer cuando las personas en roles directivos procuran resolver este mandato por la vía de acciones o programas puntuales.

Por último, quedan las siguientes preguntas: ¿es posible la multiculturalidad y la interculturalidad en el discurso académico? ¿La academia prefiere continuar la matriz colonial que no da lugar a otras formas de pensarse un lugar en el mundo? ¿Es posible una educación para todos, o tenemos los pueblos indígenas que construir universidades propias 
que nos permitan pensar nuestra cultura y tradiciones? ¿Las comunidades indígenas pueden hacer ciencia para sí mismas? Y de forma más específica, a partir de los resultados de este ejercicio, nuevas inquietudes sobre: ¿cómo se asumen los juegos ancestrales de los pueblos originarios en los procesos de formación profesional en el campo de la Educación Física? y ¿cómo funcionan los juegos ancestrales como propuesta estratégica del diálogo intercultural en los currículos? Interrogantes surgidas en el desarrollo de la investigación que deben tenerse en cuenta, a nuestro modo de ver, para dar continuidad a la línea de profundización de la diversidad.

\section{Referencias}

Bardin, L. (1986). Análisis de contenido. Madrid, España: Akal.

Bracht, V. y Crisorio, R. (2003). La educación física en Brasil y Argentina. Río de Janeiro, Brasil: Conselho.

Castro, S. (2007). Decolonizar la universidad. La hybris del punto cero y el diálogo de saberes. En S. Castro-Gómez y R. Grosfoguel (Eds.). El giro decolonial. Reflexiones para una diversidad epistémica más allá del capitalismo global (pp. 79-91). Bogotá, Colombia: Pontificia Universidad Javeriana - Instituto Pensar, Universidad Central - IESCO, Siglo del Hombre.

Corona, S. y Kaltmeier, O. (2012). En diálogo. Metodologías horizontales en ciencias sociales y culturales. Barcelona, España: Gedisa.

Constitución política de Colombia de 1991 (2016). Bogotá, Colombia: Editorial Unión.

De Sousa, B. (2010). Descolonizar el saber, reinventar el poder. Montevideo, Uruguay: Ediciones Trilce - Extensión universitaria, Universidad de la República.

Delgado, J. (1998). Métodos y técnicas cualitativas de investigación en ciencias sociales. Madrid, España: Síntesis. 
INTERCULTURALIDAD EN LOS PROCESOS DE FORMACIÓN PROFESIONAL EN EDUCACIÓN FÍSICA EN

COLOMBIA / HURTADO-CERÓN, MOLINA-BEDOYA, OSORIO-LINARES

Gavilán, V. (2011). El pensamiento en espiral: el paradigma de los pueblos indígenas. Santiago, Chile: Ñuke Mapuförlaget.

Geertz, C. (2003). La interpretación de las culturas. Barcelona, España: Gedisa.

Ley 181. Ley del deporte, la recreación, el aprovechamiento del tiempo libre y la Educación Física y se crea el Sistema Nacional del Deporte. Diario Oficial 41.679, Congreso de Colombia, 18 de enero de 1995. Recuperado de http://www.alcaldiabogota.gov.co/sisjur/normas/Norma1.jsp?i=3424

Ley 30. Ley de Educación Superior. Diario Oficial 40.700, Congreso de Colombia, 28 de diciembre $\quad 1992 . \quad$ Recuperado de http://www.alcaldiabogota.gov.co/sisjur/normas/Norma1.jsp?i=253

Maura, V. G. y Tirados, R. M. (2008). Competencias genéricas y formación profesional: un análisis desde la docencia universitaria. Revista Iberoamericana de Educación, (47), 185-209.

Ministerio de Educación Nacional (2000). Serie lineamientos curriculares. Educación Física, Recreación $\quad y \quad$ Deporte. Recuperado de http://www.mineducacion.gov.co/1759/articles-339975_recurso_10.pdf

Ministerio de Educación Nacional (2010). Orientaciones pedagógicas para la Educación Física, Recreación $\quad$ y Deporte. Recuperado de http://www.mineducacion.gov.co/1759/articles340033_archivo_pdf_Orientaciones_ EduFisica_Rec_Deporte.pdf

Silva, A. M. y Molina, V. (2015). Formación profesional en Educación Física en América Latina. Encuentros, diversidades y desafíos. Jundiaí-SP, Brasil: Paco Editorial.

Silva, A. M. y Molina, V. (2017). Educación Física en América Latina. Currículos y horizontes formativos. Jundiaí-SP, Brasil: Paco Editorial.

Sandoval, E. (2016). Educación indígena zapatista para la paz y la no-violencia. Espacio Abierto: Cuaderno Venezolano de Sociología, 25(1), 23-36. 
REVISTA SABERES EDUCATIVOS, No 4, ENERO-JUNIO 2020

Sierra, Z. (2004). Voces indígenas universitarias: expectativas, realidades y sueños. Medellín, Colombia: Marín Vieco.

Vargas, I. (2012). La entrevista en la investigación cualitativa: nuevas tendencias y retos. Calidad en la Educación Superior, 3(1), 119-139.

Villodre, B. (2012). Pluriculturalidad, multiculturalidad e interculturalidad, conocimientos necesarios para la labor docente. Hekademos, 1(11), 67-76.

Walsh, C. (2008). Interculturalidad, plurinacionalidad y decolonialidad: las insurgencias político- epistémicas de refundar el Estado. Tabula Rasa, (9), 131-152. 